\title{
Optimization of Urban Cold Chain Transport Routes under Time-Varying Network Conditions
}

\author{
Shouchen Liu $\mathbb{D}^{1,2}$ and Cheng Zhang $\mathbb{D}^{1}$ \\ ${ }^{1}$ School of Transportation and Logistics, East China Jiao Tong University, Nanchang 330013, China \\ ${ }^{2}$ School of Business Administration, Fujian Business University, Fuzhou 350012, China \\ Correspondence should be addressed to Shouchen Liu; 2019019082300004@ecjtu.edu.cn
}

Received 20 September 2020; Revised 26 January 2021; Accepted 9 March 2021; Published 23 March 2021

Academic Editor: Eneko Osaba

Copyright (c) 2021 Shouchen Liu and Cheng Zhang. This is an open access article distributed under the Creative Commons Attribution License, which permits unrestricted use, distribution, and reproduction in any medium, provided the original work is properly cited.

\begin{abstract}
Given the time-efficient characteristics of urban cold chain transportation and the time-varying characteristics of urban road speed, customers encounter the problem of limited vehicle path optimization due to a fuzzy time window. An optimization model of urban cold chain transportation with the objective function as the minimum total cost is constructed under the premise of service reliability, and an artificial immune particle swarm optimization algorithm is designed to solve the model. For an empirical analysis of Xiamen's cold chain transportation, a two-stage solution involving "static optimization and dynamic optimization" is used to verify the effectiveness of the model and the practical value of this research. Results show that the time-varying model can effectively reflect the situation of urban road transportation and satisfy the timeliness requirement of urban cold chain transportation.
\end{abstract}

\section{Introduction}

With the advancement of the economy and the improvement of people's living standards, China's cold chain logistics has developed rapidly, and the requirements for cold chain distribution are constantly improving. The traditional cold chain distribution with large quantities and few batches cannot meet the current distribution demand, and smallbatch, multi-frequency, multi-variety cold chain distribution has become the primary trend in logistics distribution. Given that most of the roads in cities are time varying, urban cold chain transport links have become increasingly complex and random. Therefore, the optimization of urban cold chain transportation paths should consider the transportation cost, loss of value, and decrease in service reliability caused by timeliness. In the process of cold chain distribution, the product quality gradually decreases with the passage of time, which not only causes damage to cold chain logistics enterprises, but also reduces customer experience. This urgent problem in the industry needs to be solved.

Domestic and foreign research on cold chain logistics vehicle routing problems focused on the optimization of cold chain logistics transport routing with time window constraints and optimization of cold chain logistics vehicle routing with multiple distribution centers, multiple vehicle models, and the lowest carbon emission. The research on cold chain delivery time windows is as follows. Govindan et al. [1] constructed a multi-objective optimization model for the integrated decision and allocation of perishable goods in a supply network with a time window. Xiao et al. [2] analyzed the cost of each link in cold chain distribution and constructed a VRP model with a minimum assembly in consideration of various constraints, such as vehicle load and time window. Kang et al. [3] considered vehicle use, transportation, cargo damage, refrigeration, penalty, and carbon emission costs as total cost optimization objectives to optimize the distribution path of cold chain logistics. Some scholars have also conducted researches on multi-distribution centers and multi-model cold-chain logistics vehicle routes: Tarantilis et al. [4] examined the distribution of meat and milk in Greece, studied the route problem of open cold chain logistic distribution vehicles with multiple vehicle types and distribution centers, and solved the problem with the threshold value method. Morim et al. [5] considered the 
constraints of multiple time windows and vehicle models and built a vehicle distribution path optimization model. Rabbani et al. [6] studied the deterministic vehicle routing problem under multiple intermediate warehouses and proposed the concept of perishable food freshness to obtain the optimal distribution path. Zhang et al. [7] designed a mathematical model of a cold chain logistic vehicle path with multiple vehicle fields and models and solved it by using the genetic algorithm and elite selection method. These studies have a certain reference value, but they mainly emphasize economic goals and the influence of the external environment on the speed of the vehicle is ignored. However, with the continuous increase in urban traffic load, urban traffic congestion has become a normal state, and the passage time of road segments changes constantly with time. The vehicle routing problem based on static traffic networks is different from the actual situation. A static model often takes the solution with a short distance but long transportation time as the optimal solution, which is contrary to the objective of cold chain logistics decision-makers to pursue timeliness. A time-varying network can address the fluctuation of urban cold chain transport vehicle speed as realistically as possible, which is close to the actual situation. Considering the time-varying vehicle path has become a research hotspot in recent years, in terms of traffic congestion, Zheng [8] added traffic congestion factors to their models when solving the cold chain logistics distribution vehicle path and, respectively, used an improved genetic algorithm and a stage optimization method to solve the problem. Lan et al. [9] built a vehicle path optimization model that considers urban road congestion and used a hybrid genetic algorithm to solve the model. Deng et al. [10] built a mixed-integer optimization model for military logistics distribution with a time window and used the genetic algorithm to solve the model. Wang et al. [11] studied the green vehicle routing problem with capacity constraints and used competitive meme algorithm to solve the model.

Scholars have also conducted research on the timevarying characteristics of urban road networks, which lead to the uncertainty of the travel time of cold chain transport vehicles. They mainly studied the shortest problem with time variation, such as section impedance (trip time or trip cost) and usually used deterministic time-dependent functions to represent section impedance. Yang et al. [12] built the simulated annealing algorithm to solve the vehicle path problem under time-varying conditions. Li et al. [13] proposed a new method to deal with the general time-varying travel agent problem and conducted modeling and solution derivation. Li [14] discussed a class of travel planning problems under time-varying conditions. Shi et al. [15] analyzed the time-varying characteristics of a transport network, built a simulation model under time-varying conditions, and used a hybrid genetic algorithm to solve the model. Zhang et al. [16] examined the scheduling problem of product distribution vehicles in a multi-temperature area under a time-varying road network environment, established a mathematical optimization model with a minimum total distribution cost, and used the solution model of the simulated annealing algorithm.

In summary, existing research results provide a good basis for the in-depth study of urban cold chain transportation under time-varying network conditions. However, there are still the following deficiencies. First, existing literature concentrated on the two-layer, timevarying network problem. Second, most existing studies focused on the mathematical model and solving algorithm of the vehicle path optimization problem in an urban road static network. The research on vehicle path optimization in an urban road dynamic network focused on road congestion avoidance and the shortest problem with time variation, such as section impedance (trip time or trip cost). In view of these research deficiencies, the main contributions of the paper are summarized as follows: (1) the urban cold chain transport path model constructed in this study involves a three-layer network, that is, supplier-distribution centercustomer. (2) The real-time situation of urban roads is reported based on the big traffic data of Baidu smart map (China Urban Congestion Index), and the dynamic vehicle path optimization model and solving algorithm under urban time-varying network conditions are established to provide a decision-making reference for cold chain enterprises as they implement urban cold chain transportation.

The rest of the paper is organized as follows. Section 2 presents the analysis and hypothesis of urban cold chain transportation under time-varying network conditions and introduces the cold chain vehicle travel time analysis. Section 3 take the lowest total cost as the objective function to establish an urban cold chain transportation model under the condition of time-varying traffic network conditions. In Section 4, an artificial immune particle swarm optimization (AI-PSO) model is designed. Section 5 presents the design of Xiamen cold chain transportation as an empirical analysis, uses the optimization algorithm to solve the case in two stages of "static optimization" and "dynamic optimization," and discusses the calculation results. The article is summarized in Section 6.

\section{Problem Description and Assumptions}

The urban cold chain transportation network involves suppliers, distribution centers, and customers. The distribution centers collect the demand information of customers and send this information to the suppliers. The supplier needs to meet time window requirements to provide service to the distribution centers. The distribution centers also need to meet time window requirements to provide delivery service to the customer, and time penalty costs are incurred for any deviation from the time window. The driving route of the supplier and distribution centers is determined. On the premise of satisfying the target of distribution centers and providing customer satisfaction, the required distribution business is completed with the lowest total cost and the least deviation from the time window.

\subsection{Model Assumptions}

(1) One supplier, multiple distribution centers, and multiple customers are located in different regions of the same city. 
(2) With regard to the supply of distribution center, no commodity allocation exists between distribution centers.

(3) With regard to the supply of customers by the distribution centers, no commodity allocation exists between customers.

(4) When the vehicle is not fully loaded, the commodity demand required by the distribution center is less than the carrying capacity of the supplier's vehicle. A vehicle can complete the distribution tasks required by multiple distribution centers. The demand for goods required by stores is smaller than the carrying capacity of vehicles in the distribution center, and one vehicle can complete the distribution tasks required by multiple stores.

(5) Suppliers adopt a vehicle distribution strategy for distribution center or store service; that is, each distribution center or store can be serviced by multiple distribution vehicles, and all service points can be served.

(6) The delivery vehicles have different models, and the delivery task on each line is only undertaken by one vehicle.

(7) The freight rate per unit of the distribution vehicle is independent of the vehicle type and is the same. Time-varying events, such as accidents, traffic jams, weather conditions, and other emergencies, that occur during the journey should be considered.

(8) It is assumed that the statistical law $o$ of the time of vehicles passing through each section in the traffic network is known; the time of vehicles passing through section $(O, D)$ follows the normal distribution $N\left(\mu_{\mathrm{OD}}, \sigma\right)$.

2.2. Travel Time Analysis of Cold Chain Vehicles under TimeVarying Network Conditions. Suppose that $m$ parallel paths exist between an $O D$ pair in the urban traffic network. Path length $D_{i j}$ and driving speed $V_{i}$ affect the travel time of each path $t_{(y)}$, and congestion coefficient $\beta$ directly affects driving speed $V_{i}$. Owing to the time-varying characteristics of the urban traffic network, a sudden traffic event occurs at point $A$ when the vehicle travels to point Ton a certain $O D$ path $i$, as shown in Figure 1.

The capacity of $T A$ of the road section will inevitably decrease, resulting in a change in vehicle travel time from $t_{(y)}$ to $t_{(s)}$. The travel time function of route $i$ is

$$
\begin{aligned}
t_{(y)}= & t_{(y)} \mathrm{OT}+t_{(y)} T A+t_{(y)} A D=t_{(y)} \mathrm{OT} \\
& +t_{(s)} T A+t_{(y)} A D .
\end{aligned}
$$

If similar situations occur in multiple $O D$ sections, then

$$
t_{(y)}=\sum t_{(y)}+\sum t_{(s)} .
$$

If $t_{(y)}$ is the driving time of vehicles on road $r$ without congestion, then

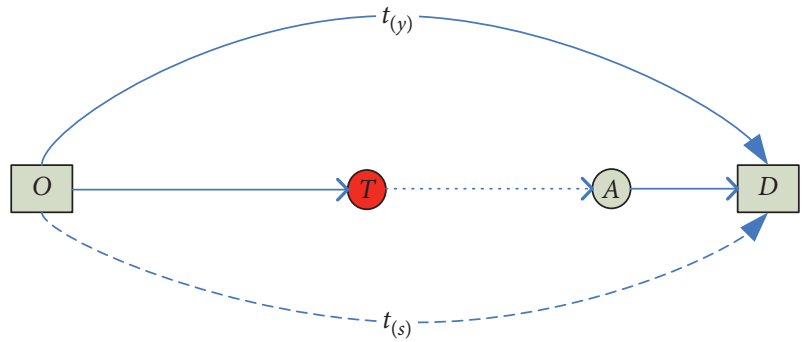

Figure 1: Diagram of the network of traffic emergencies encountered during vehicle driving.

$$
t_{(y)}=\frac{L_{i r}}{V_{i r}},
$$

where $L_{i r}$ represents the length of section $r$ on route $i$ without congestion $\forall i ; r$ and $V_{i r}$ represents the speed at which no congested section $i$ exists on route $r, \forall i ; r$.

Assuming that the vehicles can reach normal driving speed $V_{i}$ on a road without congestion, steering coefficient $\mu$ is used to measure the actual driving difficulty of the road. Therefore, vehicle speed $V_{i r}$ is related to the number of left and right turns and the number of crossings.

$$
\begin{aligned}
\mu & =\frac{\left(3 \mu_{L}+\mu_{R}+2 \mu_{C}\right)}{L_{i r}}, \\
V_{\text {ir }} & =V_{i} \times(1-0.1 \mu),
\end{aligned}
$$

where $\mu$ represents the steering coefficient, $\mu_{L}$ is the number of left turns, $\mu_{R}$ represents the number of right turns, and $\mu_{C}$ is the number of crossings [17].

Assuming that the vehicle's driving speed in the congested section $m$ is $V_{i m}$, the vehicle's driving speed $V_{i m}$ is related to the times of crossing and congestion coefficient $\beta$.

$$
V_{i m}=V_{i} \times(1-0.1 \mu) \times \beta,
$$

where congestion coefficient $\beta$ is inversely proportional to the capacity of path $i$. This study assumes that congestion coefficient $\beta$ is related to accident $\theta_{1 h}$, traffic jam $\theta_{2 h}$ (in this paper, traffic congestion is assumed to be the inverse of the real-time traffic congestion index), vehicle performance $\theta_{3 h}$, weather condition $\theta_{4 h}$, and other emergencies $\theta_{5 h}$. Their influence on speed is divided into five grades: normal $(h=1)$, mild $(h=2)$, medium $(h=3)$, severe $(h=4)$, and very severe $(h=5)$, i.e., $\beta=\theta_{1 h} \times \theta_{2 h} \times \theta_{3 h} \times \theta_{4 h} \times \theta_{5 h}$, $h \in\{1,2, \ldots, 5\}$.

\section{Building the Model}

The reliability of cold chain distribution service is an indicator of the service level of urban cold chain logistics. In the process of urban cold chain logistics operation, the cold chain logistics distribution network is the core to improve the reliability of urban cold chain service, so it is particularly important to choose the appropriate cold chain logistics center and scientific transportation network. 
3.1. Service Reliability Analysis. A customer satisfaction model is constructed based on supply chain theory. Service reliability is a function of service time $t$. The fuzzy number is a convex fuzzy set defined in the real number domain, and commonly used fuzzy number functions include triangular and trapezoidal fuzzy numbers. The membership functions of triangular and trapezoidal fuzzy numbers are shown in Figure 2.

Trapezoidal fuzzy number $t_{k}=\left(e_{k}, e_{k}^{\prime}, l_{k}^{\prime}, l_{k}\right)$ is adopted in this study. Customer $B i$ receives the highest reliability of cold chain material service within time window $\left(e_{k}^{\prime}, l_{k}^{\prime}\right)$, and the quantization is 1 . The receipt of cold chain supplies within $\left(e_{k}, e_{k}^{\prime}\right)$ or $\left(l_{k}^{\prime}, l_{k}\right)$ is acceptable, but the satisfaction decreases with time. When time window $\left(e_{k}, l_{k}\right)$ is exceeded, the satisfaction is quantified as 0 . Therefore, the time $t_{k}$ for customer $B i$ to receive the goods and materials is expressed as $\eta\left(t_{k}\right)$ by the membership function of the trapezoidal fuzzy number.

$$
\eta\left(t_{k}\right)= \begin{cases}0, & t_{k}<e_{k}, \\ \frac{t_{k}-e_{k}}{e_{k}^{\prime}-e_{k}}, & e_{k} \leq t_{k}<e_{k}^{\prime}, \\ 1, & e_{k}^{\prime} \leq t_{k} \leq l_{k}^{\prime}, \\ \frac{l_{k}-t_{k}}{l_{k}-l_{k}^{\prime},} & l_{k}^{\prime}<t_{k} \leq l_{k}, \\ 0, & t_{k}>l_{k} .\end{cases}
$$

On the basis of the membership function of trapezoidal fuzzy number $t_{k}=\left(e_{k}, e_{k}^{\prime}, l_{k}^{\prime}, l_{k}\right)$, the optimization objective of transportation time is transformed into the optimization objective of service reliability. Considering the particularity of urban cold chain logistics, customer $B i$ receives cold chain materials within the specified time window. When the time is earlier than $e_{k}$, the vehicle needs to wait, resulting in waiting costs. If the time is later than $l_{k}$, sales will be affected, resulting in opportunity loss. However, in the process of the vehicle, accidents, vehicle performance, traffic, weather conditions, or other emergencies (e.g., time-varying factors) affect vehicle moving speed, and vehicle traffic time changes from $t_{(y)}$ to $t_{(s)}$, leading to the delayed delivery of cold chain goods delivered to the customer. This delay affects the vehicle utility curve, and the service reliability curve changes. If the service reliability maximization goal is not achieved, then the model will be re-analyzed, and real-time optimization of the vehicle travel path will be performed.

Except for cold chain distribution, the reliability of other cold chain logistics links in the cold chain logistics operation is assumed to be 1 . Cold chain distribution factors affect the reliability of the entire cold chain logistics service. The reliability of the service of the cold chain logistics center refers to the probability of delivering cold chain products to customers within a specified time under certain conditions $\left(P_{(s) i j}\right)[18]$ :

$$
\begin{aligned}
P_{(s) i j} & =p\left(e_{k} \leq t_{(s) i j} \leq l_{k}\right), \\
& =p\left(\frac{e_{k} \leq d_{i j}}{V_{(s) i j} \leq l_{k}}\right), \\
& =p\left(\frac{d_{i j}}{l_{k}} \leq V_{(s) i j} \leq \frac{d_{i j}}{e_{k}}\right)=1-F V_{i j}\left(\frac{d_{i j}}{l_{k}}, \frac{d_{i j}}{e_{k}}\right) .
\end{aligned}
$$

Then, according to formula (7), the calculation formula of the reliability of the distribution center for multiple customer services is

$$
\begin{aligned}
P_{(s)} & =\frac{\sum_{i \in M} \sum_{j \in M} Q_{i j} P_{(s) i j}}{\sum_{i \in M} \sum_{j \in M} Q_{i j}} \\
& =\frac{\sum_{i \in M} \sum_{j \in M} Q_{i j}\left[1-F V_{i j}\left(d_{i j} / l_{k}, d_{i j} / e_{k}\right)\right]}{\sum_{i \in M} \sum_{j \in M} Q_{i j}} .
\end{aligned}
$$

For conciseness, the representation of the reliability of cold chain logistics services in this model is presented in Table 1.

3.2. Optimization Model of Urban Cold Chain Transport Path under Time-Varying Network Conditions. Assuming that $G$ is a set of suppliers, $g=1, \ldots, f . M$ is a set of cold chain distribution centers $i=1, \ldots, m$. $N$ is the customer set, $j=1, \ldots, n$. Suppliers who import cold chain goods need to supply $n$ customers through the cold chain logistics distribution center after storage and cooling distribution. Static and dynamic optimization are considered in accordance with the time window requirements of each distribution center and customer. The model is as follows: supplier (dock) $\longrightarrow$ distribution center $\longrightarrow$ customer (Figure 3). First, the distribution center is determined. A total of $m$ alternative distribution centers exist, and $m_{0}$ needs to be determined from the alternative distribution centers. Second, the customers of the distribution center are determined, and the transportation route from the supplier (terminal) to the distribution center is planned. Third, the transportation route from the distribution center to the customer is set to guarantee the lowest total cost.

3.2.1. Model Parameters. The symbols of the urban cold chain vehicle path optimization model are summarized in Table 2. 


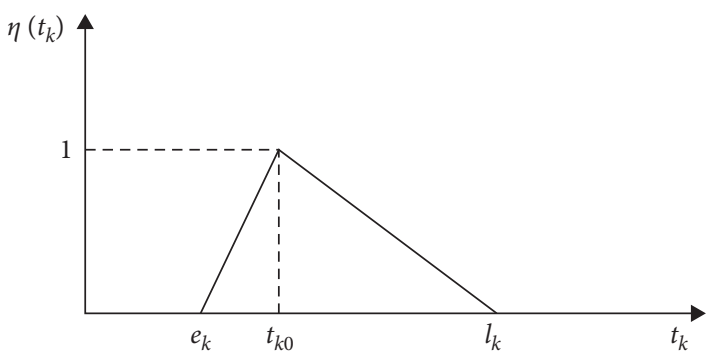

(a)

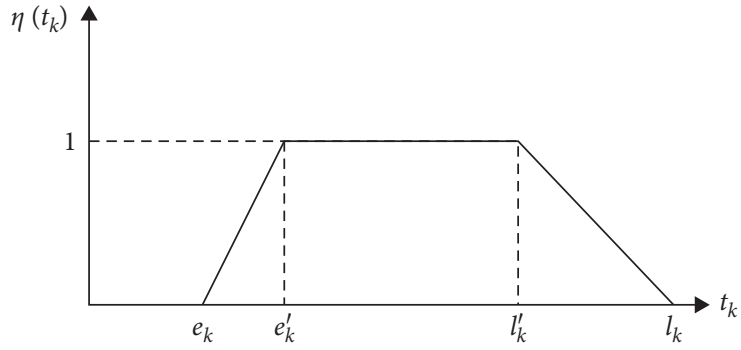

(b)

FIGURE 2: Membership function diagram of triangular and trapezoidal fuzzy numbers. (a) Graph of triangular fuzzy membership function. (b) Diagram of trapezoidal fuzzy membership function.

TABLE 1: Symbol of logistics service reliability.

\begin{tabular}{lc}
\hline Notation & Definition \\
\hline$d_{i j}$ & The distance between distribution center $i$ and customer $j$ \\
$V_{i j}$ & The travel speed from distribution center $i$ to customer $j$ \\
$F V_{i j}$ & $\begin{array}{c}\text { The distribution function of driving speed from distribution center } i \text { to customer } j . \text { Driving speed is affected by accidents, } \\
\text { weather conditions, traffic jam, vehicle performance, and other emergencies, and travel speed conforms to the normal } \\
\text { distribution }\end{array}$ \\
$P_{(s)}$ & The reliability of logistics service in the distribution center \\
$P_{(s) i j}$ & The reliability of distribution center $i$ in terms of providing logistics service to customer $j$
\end{tabular}

$$
\begin{aligned}
& \operatorname{Min} C_{1}=\sum_{g=1}^{f} \sum_{i=1}^{m} Z_{g i} e_{g i} c_{g i} Q_{g i}+\sum_{g=1}^{f} \sum_{i=1}^{m} Z_{g i} \sum_{j=1}^{n} c_{i j} b_{i j} Q_{i j} d_{i j}+\sum_{g=1}^{f} \sum_{i=1}^{m} Z_{g i} Q_{g i} \theta p+\frac{\sum_{i=1}^{m} f_{i}}{30}+\sum_{i=1}^{m} v_{i}\left(w_{i}\right)^{\varphi} \\
& +\gamma_{1} \sum_{i=1}^{m} \max \left(A_{i}-T_{i}, 0\right)+\gamma_{2} \sum_{i=1}^{m} \max \left(T_{i}-B_{i}, 0\right)+\gamma_{3} \sum_{j=1}^{n} \max \left(a_{j}-t_{j}, 0\right)+\gamma_{4} \sum_{j=1}^{n} \max \left(t_{j}-b_{j}, 0\right)+\delta t_{z}, \\
& \text { s.t. } t_{z}=\sum_{g=1}^{f} \sum_{i=1}^{m} Z_{g i}\left(\left(t_{(y) g i} \vee t_{(s) g i}\right)+Q_{g i} t_{0}\right)+\sum_{g=1}^{f} \sum_{i=1}^{m} Z_{g i} \sum_{j=1}^{n}\left(\left(t_{(y) i j} \vee t_{(s) i j}\right)+Q_{i j} t_{0}\right) \text {, } \\
& \sum_{g=1}^{f} \sum_{i=1}^{m} Z_{g i}=m_{0} \\
& \sum_{i=1}^{m} \sum_{j=1}^{n} b_{i j}=n \\
& \sum_{i=1}^{n} b_{i j}=1, \quad \text { where }: j=1,2, \ldots, n \\
& \sum_{g=1}^{f} \sum_{i=1}^{m} Z_{g i} \sum_{j=1}^{n} b_{i j}=n, \\
& \sum_{g=1}^{f} \sum_{i=1}^{m} Z_{g i} \sum_{j=1}^{n} b_{i j} Q_{i j}=\sum_{j=1}^{n} Q_{j} \text {, } \\
& \sum_{i=1}^{m} \sum_{j=1}^{n} b_{i j} Q_{i j} \leq Q_{0}, \quad \text { where }: i=1,2, \ldots, m ; j=1,2, \ldots, n
\end{aligned}
$$




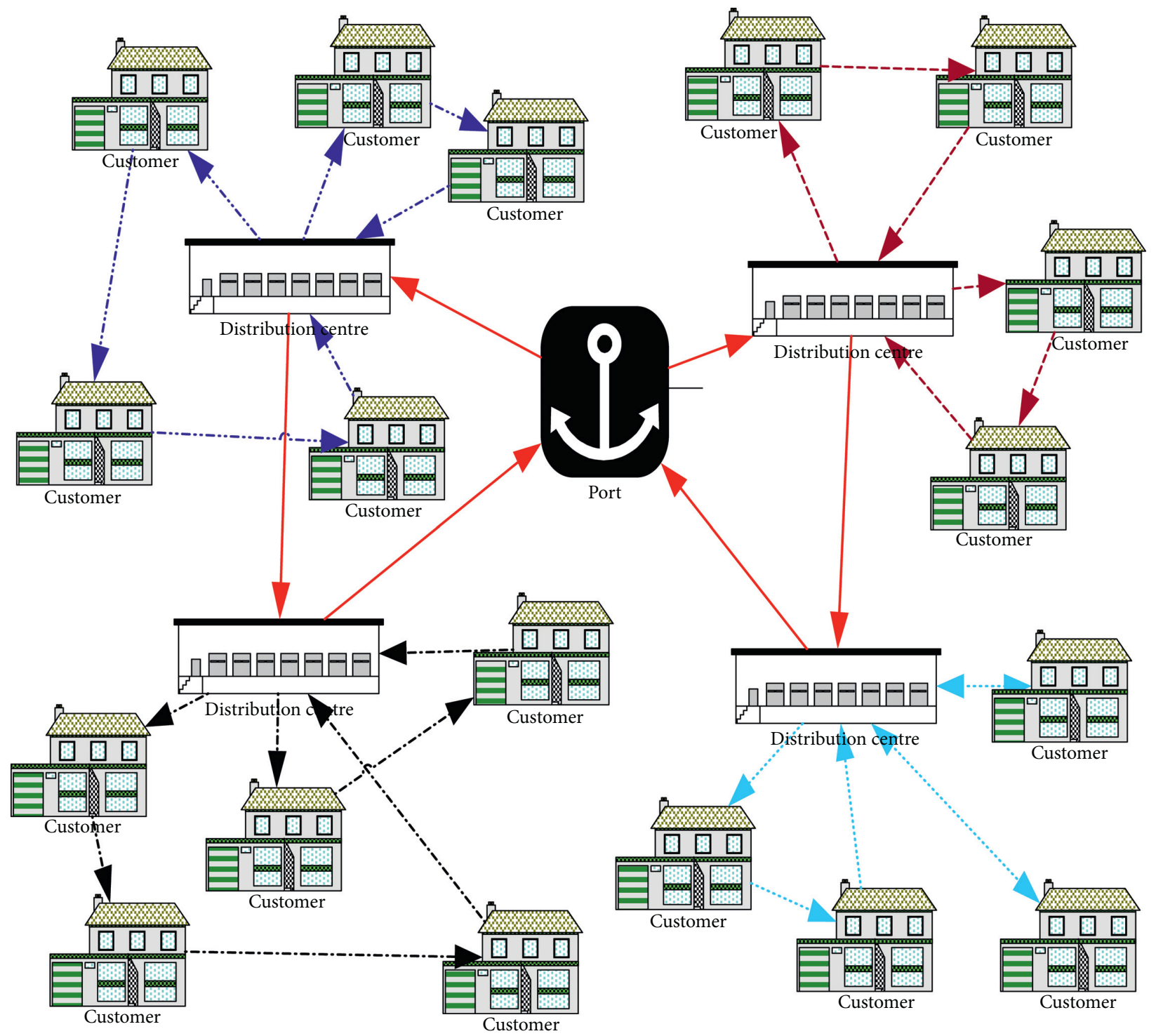

Figure 3: Topology diagram of supplier-distribution centers-customer network.

$$
\begin{aligned}
\sum_{g=1}^{f} \sum_{i=1}^{m} Z_{g i}-\sum_{j=1}^{n} b_{i j} & \leq 0, \quad \text { where }: i=1,2, \ldots, m, \\
t_{(y) g i} & =\frac{e_{g i}}{v_{(y) g i}}, \\
t_{(s) g i} & =\frac{e_{g i}}{v_{(s) g i}}, \\
t_{(y) i j} & =\frac{d_{i j}}{v_{(y) i j}}, \\
t_{(s) i j} & =\frac{d_{i j}}{v_{(s) i j}},
\end{aligned}
$$


TABLE 2: Notation for the model.

\begin{tabular}{|c|c|}
\hline Notation & Definition \\
\hline$Q_{q i}$ & The volume of transportation from supplier $g$ to distribution center $i$ \\
\hline$Q_{i j}$ & The volume of delivery from distribution center $i$ to customer $j$ \\
\hline$c_{g i}$ & The unit freight rate from supplier $g$ to distribution center $i$ \\
\hline$c_{i j}$ & The unit freight rate of the product from distribution center $i$ to customer $j$ \\
\hline$e_{g i}$ & The distance between supplier $g$ and distribution center $i$ \\
\hline$d_{i j}$ & The distance between distribution center $i$ and customer $j$ \\
\hline$Q_{0}$ & The upper limit of the quantity distributed by the distribution center \\
\hline$f_{i}$ & The cost of leasing or building the distribution center in $i$ places \\
\hline$v_{i}$ & The unit variable cost of distribution center $i$ \\
\hline$w_{i}$ & The actual transfer quantity of distribution center $i$ \\
\hline$t_{0}$ & The unit laytime \\
\hline$T_{i}$ & The time when the transport vehicle arrives at distribution center $i$ \\
\hline$A_{i}$ & The earliest service time of distribution center $i$ \\
\hline$B_{i}$ & The latest service acceptance time of distribution center $i$ \\
\hline$t_{j}$ & The time when the vehicle arrives at customer $j$ \\
\hline$a_{j}$ & The earliest time for the customer to accept the service \\
\hline$b_{j}$ & The latest time for the customer to accept the service \\
\hline$\gamma_{1}$ & The unit penalty cost of reaching the distribution center $i$ earlier than $A_{i}$ \\
\hline$\gamma_{2}$ & The unit penalty cost of arriving at distribution center $i$ later than $B_{i}$ \\
\hline$\gamma_{3}$ & The unit penalty cost of reaching customer $j$ earlier than $a_{j}$ \\
\hline$\gamma_{4}$ & The unit penalty cost for reaching customer $j$ later than $b_{j}$ \\
\hline$\delta$ & The consumption of vehicle refrigeration cost per unit time \\
\hline$P$ & The average unit value of cold chain goods \\
\hline$\theta$ & The coefficient of spoilage rate in the transportation of cold chain goods \\
\hline$q$ & The capacity ceiling of the construction or leasing of the cold chain distribution center \\
\hline$v$ & $v$ is the average speed of imported fruit cold chain distribution vehicles \\
\hline$h$ & The unit freight of imported fruit \\
\hline
\end{tabular}

$$
\begin{aligned}
& Z_{g i}= \begin{cases}1, & \text { indicates that the } g-\text { th supplier delivers to the } i-\text { th cold chain distribution center, } \\
0, & \text { otherwise }\end{cases} \\
& b_{i j}= \begin{cases}1, & \text { the } i-\text { th cold chain distribution center to the } j-\text { th customer, } \\
0, & \text { otherwise. }\end{cases}
\end{aligned}
$$

\subsubsection{Urban Cold Chain Transport Path Optimization Model.} The model is built as follows.

Formula (9) is the objective function that represents the minimum total cost of cold chain goods from the supplier to the customer through the distribution center. Formula (10) presents the total time required for the cold chain goods to be transported from suppliers to customers. Formula (11) indicates that $m_{0}$ cold chain distribution centers are selected from $m$ alternative cold chain distribution centers. Formula (12) represents the supply and demand relationship of $n$ pairs between the cold chain distribution center and the customer. Formula (13) shows that each customer can only be served by a single cold chain distribution center. Formula (14) indicates that, for each cold chain distribution center selected, the corresponding distribution relationship is limited. Formula (15) shows that the distribution amount from the supplier to the cold chain distribution center is equal to the sum of the cold chain distribution center to customer delivery volume. Formula (16) indicates that the total delivery quantity of each cold chain distribution center does not exceed the upper limit $Q_{0}$. Formula (17) represents the corresponding customer of the cold chain distribution center, and in all cases, a cold chain distribution center is selected and customers are present. Formula (18) represents the estimated driving time from supplier $g$ to cold chain distribution center $i$. Formula (19) represents the estimated driving time from cold chain distribution center $i$ to customer $j$. Formula (20) denotes the actual driving time from supplier $g$ to cold chain distribution center $i$. Formula (21) provides the actual driving time from cold chain distribution center $i$ to customer $j$. Formulas (21) and (23) are decision variables.

\section{Optimization Algorithm Design}

In this paper, the decomposition method is used to decompose the urban cold chain transportation path optimization problem into two interconnected sub problems. First, this paper solves the problem of customer attribution positioning. Then, the optimal solution of the second subproblem is based on the optimal solution of the first subproblem. Finally, according to the above solution, the objective solution of urban cold chain transportation path optimization is obtained. The problem is solved by 
MATLAB programming and artificial immune particle swarm optimization algorithm [19].

4.1. Vehicle Travel Time Calculation. In the path model with a time-varying network and symmetry, the travel time of section $\left(b_{i}, b_{j}\right)$ is expressed as $t_{(y) i j}=d_{i j} / \bar{v}_{(y) i j}$, where $\bar{v}_{(y) i j}$ is the estimated average driving speed of section $\left(b_{i}, b_{j}\right)$. However, in the time-varying network model, vehicle travel time is affected by time-varying factors, such as accidents, vehicle performance, traffic congestion, weather conditions, and other emergencies. Assuming that the departure time is $t_{0}$ and the distance is $d_{i j}$, according to Figure 1, the driving speed of vehicles is affected by time-varying factors during the driving process of vehicles on section $(O, D)[20]$. In this paper, section $(O, D)$ is divided into multiple sub-sections. If sub-section $T A$ is the $k$ segment, then the actual running speed will be $v_{(s) k i j}$, and the corresponding starting time interval will be $\left[t_{k}, t_{k+1}\right]$. The calculation flow of arrival time and travel time can be expressed as follows:

(1) Determine the value of $k$ in accordance with $t_{0} \in\left[t_{k}, t_{k+1}\right]$, obtain the corresponding $v_{(s) k i j}$, assign $d_{i j} \longrightarrow d$, and calculate $t^{\prime}=\left(t_{0}+d / v_{(s) i j}^{k}\right)$.

(2) If $t^{\prime} \leq t_{k+1}$ is true, output arrival time $t^{\prime}$ and travel time $t^{\prime}-t_{0}$, and end the loop. Otherwise, proceed to Step (3).

(3) Update $d_{i j}-v_{(s) i j}^{k}\left(t_{k+1}-t_{k}\right) \longrightarrow d$, calculate $t^{\prime}=$ $t_{k+1}+d / v_{(s) i j}^{k+1}$, update $k+1 \longrightarrow k$, and return to Step (2).

4.2. Artificial Immune Particle Swarm Optimization. In this study, artificial immune particle swarm optimization (AIPSO) is introduced to solve the proposed mathematical model. The artificial immune algorithm introduces the diversity of biological immune characteristics and immune memory into the algorithm, maintains the diversity of population particles, and can solve the problems of premature convergence, low search accuracy, and poor local search capability of the traditional immune algorithm so that the optimization results can meet practical requirements [21].

4.2.1. Production of the Initial Antibody Group. The initial antibody group is selected from the memory bank when this bank is not empty. Otherwise, the initial antibody group is generated randomly in the feasible solution space. A simple coding method is used in this study. Each site selection scheme can form an antibody of length $p$ ( $p$ represents the number of distribution centers), and each antibody represents the sequence of demand points of the selected cold chain distribution centers. This work considers a problem involving 31 customers, where $1,2, \ldots, 31$ represents the customer serial number. Four of them are selected as distribution centers. Antibody $(12,18,25,27)$ represents a feasible solution, which means that $12,18,25$, and 27 are selected as distribution centers.
4.2.2. Multiplicity Evaluation of Solutions. Suppose that the immune system consists of $a$ antibodies with a gene of length $A$.The following terms are defined:

(1) Diversity. An effective metric to measure and evaluate the differences among individuals must be developed to maintain or expand the diversity of individuals in population evolution. Average information entropy $H(a)$, which is used to measure the difference between individuals [22]. It is expressed as $H(a)=(1 / A) \sum_{i=1}^{A}$ $H_{i}(a) . H_{i}(a)$ is the information entropy of the $i$ th gene, and it is defined as $H_{i}(a)=-\sum_{j=1}^{a} p_{j i} \log _{2}^{p_{j i}}$, where $p_{j i}$ is the probability of the $j(j=1,2,3, \ldots, S)$ symbol appearing on locus $i$; that is, $p_{j i}=$ (the total number of $j$ th symbols on $i / a$ ).

(2) Similarity. The affinity between antibodies is calculated using the $R$ bit continuous method, which is a partial matching rule. First, $R$ is determined to represent the threshold of the degree of affinity. A high degree of similarity between antibodies is determined by the presence of a high $R$ or continuous $R$ bits in the two codes; otherwise, it means two different individuals. $S v, s=(k v, s / L)$, where $k v, s$ represents the same digit number of antibody $v$ and antibody $s$ and $L$ represents the length of a general antibody [23]. If the two antibodies are $(5,2,7,18,6$, $19,16,10,12)$ and $(11,8,7,26,6,19,21,9,15)$, then centers 7,6 , and 19 are the same. $k v, s=3$, and length $L$ is 9 . Therefore, the similarity degree is $1 / 3$. This similarity is calculated using the similarity in data mining [24].

(3) Antibody Concentration. This concentration refers to the proportion of similar antibodies in the population (calculated using the $R$ bit continuous method mentioned above); that is, $C v=(1 / N)$ $\sum_{j \in N} S v, s$, where $S v, s=\left\{\begin{array}{ll}1 & S v, s>T \\ 0 & S v, s<T\end{array}, T\right.$ is a predetermined threshold, $C v$ is the concentration of antibody $v, n$ is the total number of antibodies, and $N$ is the collection of antibodies [25].

(4) Expected Reproduction Rate. The expected reproduction rate of each individual in the population is determined by the affinity $(A v)$ between the antibody and the antigen and the concentration $(C v)$ of the antibody. $\quad P=a\left(A v / \sum A v\right)+(1-a)\left(C v / \sum C v\right)$, where $a$ is the diversity evaluation parameter (usually 0.95). The equation indicates that the individual concentration is inversely proportional to the expected reproduction rate, and the individual affinity is directly proportional to the expected reproduction rate.

(5) Immune Memory Unit. The addition of a certain number of antibodies with high antigen affinity in the memory unit is conducive to the updating of the memory unit. $\operatorname{DSY}\left(X_{j}\right)=\left(\sum_{i=1}^{A+a}\left|f\left(x_{j}\right)-f\left(x_{i}\right)\right| /\right.$ $\left.\sum_{j=1}^{A+a} \sum_{i=1}^{A+a}\left|f\left(x_{j}\right)-f\left(x_{i}\right)\right|\right) \quad$ and $A f=(1 / a) \sum_{i=1}^{a}$ $f\left(x_{i}\right)$, where DSY is the concentration selection probability of the antibody and $A f$ is the average fitness [26]. 
(6) Aggregate Fitness. Aggregate fitness is a modification of fitness' ${ }^{\prime}=$ fitness $\cdot \exp \left(\varphi \cdot C_{j}\right)$. The probability of antibody selection is proportional to polymeric fitness when selection is performed. When the concentration is constant, the higher the fitness is, the greater the probability of being selected is. However, when the fitness is constant, the higher the antibody concentration is, the lower the probability of selection is [27].

4.2.3. Artificial Immune Particle Swarm Optimization (AIPSO) Algorithm Flow. The AI-PSO solution to urban cold chain transport path optimization can be summarized in the following steps. The pseudocode of the AI-PSO algorithm is shown in Algorithm 1.

\section{Example Analysis}

In this paper, Xiamen Dongdu Port is used as the distribution center, and 31 customers in Xiamen Island are selected as the distribution points.

5.1. Instance-Related Data. This study uses the MATLAB program to calculate and simulate the cold chain distribution network of imported fruit in Xiamen Island. This region has one supplier (Dongdu Port) and 31 customers. In consideration of the various factors of cold chain urban transportation vehicle distribution, the service time of the model is set to 6:00 am to 12:00 am. And 6:00 am is the earliest service time of the distribution center, and the time is set as 0 . In accordance with the traffic law of Xiamen City, the traffic jam time is set from 7:00 am to 9:00 am, and the rest time is the normal driving time. Speed is related to accidents, traffic jams, weather conditions, vehicle performance, and other emergencies. AI-PSO is used to solve the urban cold chain transport optimization model, and the relevant parameters are shown in Table 3.

Four distribution centers are selected to provide distribution services to the 31 customers. The coordinate matrix of suppliers, distribution centers, and customers is shown in Table 4, and the spatial layout is shown in Figure 4. The customer's time window was obtained by investigating the cold chain operation center of imported fruit in Xiamen Free Trade (experimental) zone. If the customer is selected as a distribution center, the customer's time window is the time window for the distribution center to accept shipments from the supplier.

In this study, MATLAB R2014b programming software is used to solve the model, and the basic information of the platform is shown in Table 5. In the path optimization study, population size $M=200$ and the maximum number of iterations $\operatorname{Tmax}=100$. Memory storage capacity Overbest $=10$, cross-probability pcross $=0.5$, and mutation probability $P=0.4$. In the particle swarm algorithm, inertia weight wstart $=0.9$, wend $=0.4$, and learning factor $C_{1}=C_{2}=1.5$.
5.2. Static Optimization Phase. AI-PSO is adopted in accordance with the known information, and the time window requirements of the distribution centers and users are combined to determine the customer ownership in a static environment. The analysis results are shown in Figure 5, and the corresponding customer information is shown in Table 6.

The pre-optimization stage distribution model in a static environment is determined in accordance with the known information, and the AI-PSO solution is adopted. Supplier A services distribution centers B21, B27, B24, and B28. The total vehicle mileage is $82.173 \mathrm{~km}$, and the average service reliability of the supplier and each distribution center is 0.8787 . The five urban cold chain transportation routes, costs, vehicle driving distance, and service reliability in the static environment are shown in Table 7.

The convergence curve of AI-PSO in the static environment is shown in Figure 6, and the minimum cost of the objective function is $57,613.91$ yuan.

5.3. Dynamic Real-Time Optimization Phase. When the factors change, continued implementation of the optimization scheme in the static environment results in a failure to meet the service reliability target of the supplier or part of the distribution center. The real-time change in the congestion index from 8:00 a.m. to 18:00 a.m. on November 20, 2019, obtained with Baidu Map intelligent traffic software is shown in Figure 7. According to Baidu traffic big data, the accident coefficient of Xiamen is 0.846 , and the coefficient of other emergencies is 0.995 . The weather information in Xiamen is from China Meteorological Administration. November 20, 2019, was a sunny and cloudy with a temperature of $16^{\circ} \mathrm{C}-22^{\circ} \mathrm{C}$ and wind force of $5-6$ turning to $3-4$. The weather condition coefficient is set to 0.990 . According to the on-board database, the vehicle performance coefficient is 0.999 .

In accordance with the road characteristics in Xiamen, the driving steering coefficient is assumed to be 1. According to formula (5), the actual driving speed of the vehicle changes correspondingly. On this basis, the AI-PSO method is adopted to determine the customer ownership in the dynamic environment on the premise of meeting the requirements of the fuzzy time window. The analysis results are shown in Figure 8, and the corresponding customer information is shown in Table 8.

In accordance with the change information, a transport model of the real-time optimization stage under the condition of time-varying network conditions is built, and the AI-PSO solution is adopted. The results show that supplier A services distribution centers B16, B24, B27, and B28. The total vehicle mileage is $283.832 \mathrm{~km}$, and the average service reliability of the supplier to each distribution center is 0.8557 . The five urban cold chain transportation routes, costs, vehicle driving distance, and service reliability in the dynamic environment are shown in Table 9. The convergence curve of AI-PSO in the static environment is shown in Figure 9, and the minimum cost of the objective function is RMB 58101.23. 
Input: set population size $M=200$ and the maximum number of iterations Tmax $=100$. Overbest $=10$, cross-probability pcross $=0.5$, and mutation probability $P=0.4$. In the particle swarm algorithm, inertia weight WSTART $=0.9$, Wend $=0.4$, and learning factor $C_{1}=C_{2}=1.5$.

Output: particle position matrix $X_{n(n-1)}$, velocity matrix $V_{n(n-1)}$

Antigen recognition, fitness calculation to determine Pbest and Gbest

procedure AI-PSO

for each particle $i$

Initialize velocity $V_{i}$ and position $X_{i}$ for particle $i$

Evaluate particle I and set $P$ best $_{i}=X_{i}$

\section{end for}

Gbest $=\min \left\{\right.$ Pbest $\left._{i}\right\}$

Cluster center coding and particle clustering division are carried out;

According to the corresponding clustering division, the new clustering center is calculated and the particle fitness is updated;

while not stop

for $i=1, \ldots, n$

Initialize velocity $V_{i}$ and position $X_{i}$ for particle $i$

Evaluate particle $i$

if fit $\left(X_{i}\right)<\operatorname{fit}\left(\right.$ Pbest $\left._{i}\right)$

Pbest $_{i}=X_{i}$

if fit $\left(\right.$ best $\left._{i}\right)<$ fit $(G$ best $)$

Gbest $=$ Pbest $_{i}$

End for

End while

Print Gbest

End procedure

Algorithm 1: AI-PSO algorithm pseudocode.

TABLE 3: Setting of the correlation coefficient.

\begin{tabular}{lc}
\hline$c$ & 30 yuan $/ \mathrm{t} / \mathrm{km}$ \\
\hline$p$ & $18 \mathrm{yuan} / \mathrm{kg}$ \\
$\theta$ & 0.02 \\
$v_{(y)}$ & $30 \mathrm{~km} / \mathrm{h}$ \\
$m_{o}$ & 4 \\
$t_{o}$ & $0.25 \mathrm{~h}$ \\
$\delta$ & $100 \mathrm{yuan} / \mathrm{h}$ \\
$\gamma_{1}$ & $100 \mathrm{yuan} / \mathrm{h}$ \\
$g$ & 1 \\
$v_{i}$ & 6 yuan $/ \mathrm{t}$ \\
$f_{i}$ & $7000 \mathrm{yuan}$ \\
$Q_{o}$ & $60 \mathrm{t}$ \\
$\gamma_{2}$ & $300 \mathrm{yuan} / \mathrm{h}$ \\
$\gamma_{3}$ & $60 \mathrm{yuan} / \mathrm{h}$ \\
$\gamma_{4}$ & $200 \mathrm{yuan} / \mathrm{h}$ \\
$\varphi$ & 0.5 \\
\hline
\end{tabular}

5.4. Analysis of Experimental Results. The traditional particle swarm optimization (PSO) and mixed particle swarm optimization (GA-PSO) programs are also written using MATLAB R2014b to verify the effectiveness of the urban cold chain transport path optimization algorithm under a time-varying network. Hybrid particle swarm optimization (MSO) abandons the traditional particle swarm optimization (PSO) method of updating the particle position by tracking the extreme value, but it introduces a crossover and mutation operation in the genetic algorithm and searches for the optimal solution through the crossover between individual and population extreme values of the particle and the way of the particle's own variation. A comparison

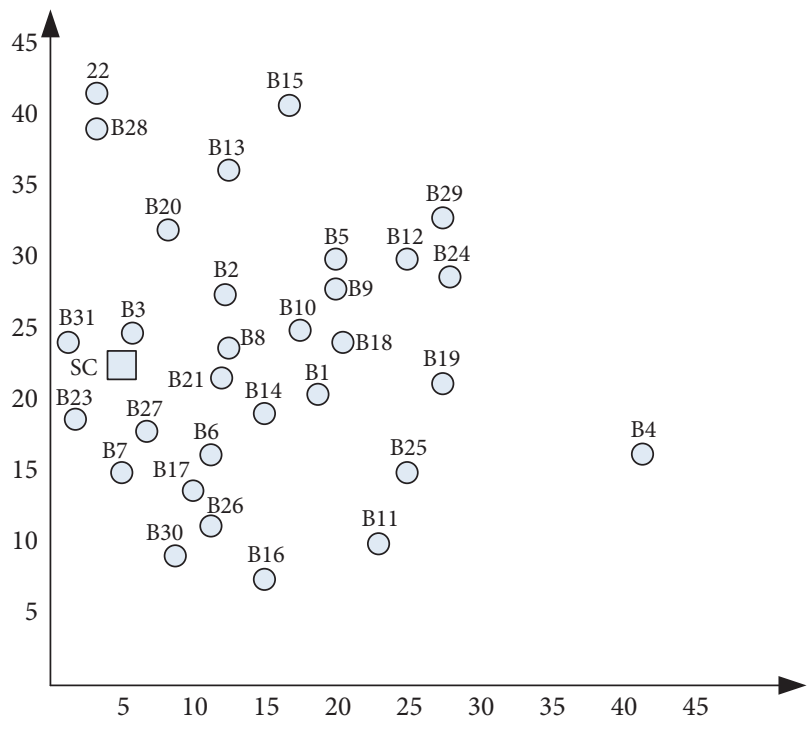

FIGURE 4: Spatial layout of suppliers and customers.

experiment is conducted with the AI-PSO algorithm designed in this paper. Under the same experimental conditions, the cold chain transport path, distance, cost, and service reliability under a static environment are calculated, and the results of the three methods are shown in Table 10. The transport path, distance, and cost of the cold chain under a dynamic environment are shown in Table 11.

As can be seen from Table 10, in the result of the twolayer model of the optimal path for vehicles under the GAPSO algorithm with urban cold chain transportation cost as 
TABLE 4: Coordinate positions of suppliers and customers, quantity demanded, and time window.

\begin{tabular}{|c|c|c|c|c|c|}
\hline Supplier & Coordinate $(\mathrm{km})$ & Customer & Coordinate $(\mathrm{km})$ & Quantity demanded (T) & Time window (MIN) \\
\hline & & B1 & $(18,21)$ & 2.44 & $(0,10,30,40)$ \\
\hline & & B2 & $(13,28)$ & 4.96 & $(0,15,50,70)$ \\
\hline & & B3 & $(6,24)$ & 3.84 & $(0,20,40,60)$ \\
\hline & & B4 & $(42,16)$ & 9.10 & $(0,10,20,30)$ \\
\hline & & B5 & $(20,30)$ & 6.31 & $(0,30,60,90)$ \\
\hline & & B6 & $(11,16)$ & 1.72 & $(0,40,90,130)$ \\
\hline & & B7 & $(5,15)$ & 5.09 & $(0,10,20,30)$ \\
\hline & & B8 & $(13,24)$ & 10.33 & $(0,15,45,60)$ \\
\hline & & B9 & $(21,28)$ & 2.53 & $(0,10,50,60)$ \\
\hline & & $\mathrm{B} 10$ & $(18,25)$ & 4.64 & $(0,10,30,40)$ \\
\hline & & $\mathrm{B} 11$ & $(23,10)$ & 5.00 & $(0,5,15,20)$ \\
\hline & & $\mathrm{B} 12$ & $(25,30)$ & 7.11 & $(0,30,60,70)$ \\
\hline & & $\mathrm{B} 13$ & $(12,36)$ & 1.69 & $(0,20,60,90)$ \\
\hline & & B14 & $(15,19)$ & 3.00 & $(0,10,20,30)$ \\
\hline & & B15 & $(16,41)$ & 3.21 & $(0,10,30,40)$ \\
\hline \multirow[t]{16}{*}{ A } & $(5,19)$ & B16 & $(15,7)$ & 4.06 & $(0,40,80,120)$ \\
\hline & & $\mathrm{B} 17$ & $(10,14)$ & 7.82 & $(0,15,30,45)$ \\
\hline & & B18 & $(23,24)$ & 9.66 & $(0,60,90,120)$ \\
\hline & & B19 & $(27,21)$ & 2.02 & $(0,15,45,60)$ \\
\hline & & $\mathrm{B} 20$ & $(8,32)$ & 5.31 & $(0,10,60,70)$ \\
\hline & & B21 & $(14,23)$ & 7.50 & $(0,25,55,80)$ \\
\hline & & B22 & $(4,42)$ & 6.00 & $(0,20,40,60)$ \\
\hline & & B23 & $(2,18)$ & 10.89 & $(0,5,20,30)$ \\
\hline & & B24 & $(27,28)$ & 4.55 & $(0,20,60,80)$ \\
\hline & & $\mathrm{B} 25$ & $(25,15)$ & 1.36 & $(0,10,30,45)$ \\
\hline & & B26 & $(11,11)$ & 5.94 & $(0,5,25,40)$ \\
\hline & & $\mathrm{B} 27$ & $(6,17)$ & 6.20 & $(0,25,55,80)$ \\
\hline & & B28 & $(7,39)$ & 4.33 & $(0,15,45,60)$ \\
\hline & & B29 & $(27,32)$ & 6.50 & $(0,20,40,60)$ \\
\hline & & B30 & $(10,10)$ & 2.44 & $(0,35,70,105)$ \\
\hline & & B31 & $(1,24)$ & 7.19 & $(0,20,80,100)$ \\
\hline
\end{tabular}

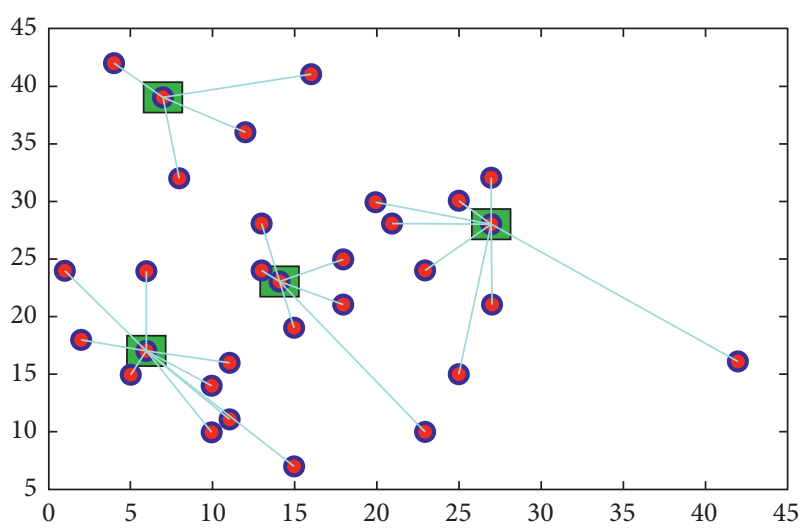

FIGURE 5: Customer ownership in a static environment.

TABLE 5: Experimental test environment.

\begin{tabular}{lc}
\hline Computer models & RedmiBook 14 \\
\hline Operating system & Windows 10 64-bit (DirectX 12) \\
CPU & Intel (R) i7-10510H CPU @ 1.80 GHz \\
Computer memory & $8 \mathrm{G} \mathrm{(DDR4} \mathrm{2666} \mathrm{MHZ)}$ \\
Hard disk & $512 \mathrm{G}$ \\
\hline
\end{tabular}

TABLE 6: Service object information of the supplier and distribution center in a static environment.

\begin{tabular}{lc}
\hline Service outlets & Service object \\
\hline A & B21, B27, B24, B28 \\
B21 & B1, B2, B8, B10, B11, B14, B21 \\
B24 & B4, B5, B9, B12, B19, B18, B24, B25, B29 \\
B27 & B3, B6, B7, B16, B17, B23, B26, B27, B30, B31 \\
B28 & B13, B15, B20, B22, B28 \\
\hline
\end{tabular}

the objective function, the supplier needs to send five A cars, one $\mathrm{C}$ car, and one $\mathrm{D}$ car to carry out distribution for the four distribution centers. The four distribution centers need to send three A cars, three B cars, one C car, and two D refrigerated transporters to distribute to 27 customers. Compared with GA-PSO and PSO, AI-PSO has differences in vehicle selection, but they are not considerable. In terms of program running time, PSO and GA-PSO require 40.1203 and $33.2169 \mathrm{~s}$, respectively, to converge. AI-PSO requires $31.3470 \mathrm{~s}$ to converge, which is $21.88 \%$ more efficient than PSO. In terms of the total cost, PSO has the lowest total cost, but its average service reliability is also the lowest (only 0.8319). The total cost and total distance of the AI-PSO algorithm are slightly increased, but the average service reliability is improved. The average service reliability of the 
TABLE 7: Cold chain transport path, distance, cost, and service reliability in a static environment.

\begin{tabular}{|c|c|c|c|c|c|}
\hline Service outlets & Shipping lines & Number and model of vehicle & Total cost & Total distance & $\begin{array}{l}\text { Service } \\
\text { reliability }\end{array}$ \\
\hline A & A-B28-B24-B21-B27-A & $5 \mathrm{~A}+1 \mathrm{C}+1 \mathrm{D}$ & 26430.624 & 82.173 & 0.9072 \\
\hline $\mathrm{B} 21$ & B21-B8-B2-B10-B1-B11-B14-B21 & $1 \mathrm{~A}+1 \mathrm{C}$ & 6730.207 & 43.493 & 0.8827 \\
\hline B27 & B27-B23-B31-B3-B6-B17-B16-B26-B30-B7-B27 & $1 \mathrm{~A}+1 \mathrm{~B}+1 \mathrm{D}$ & 8398.512 & 51.856 & 0.8196 \\
\hline B24 & B24-B29-B12-B5-B9-B18-B25-B19-B4-B24 & $1 \mathrm{~A}+1 \mathrm{~B}$ & 10631.291 & 69.102 & 0.8477 \\
\hline B28 & B28-B20-B13-B15-B22-B28 & $1 \mathrm{~B}+1 \mathrm{D}$ & 5423.274 & 35.415 & 0.9362 \\
\hline
\end{tabular}

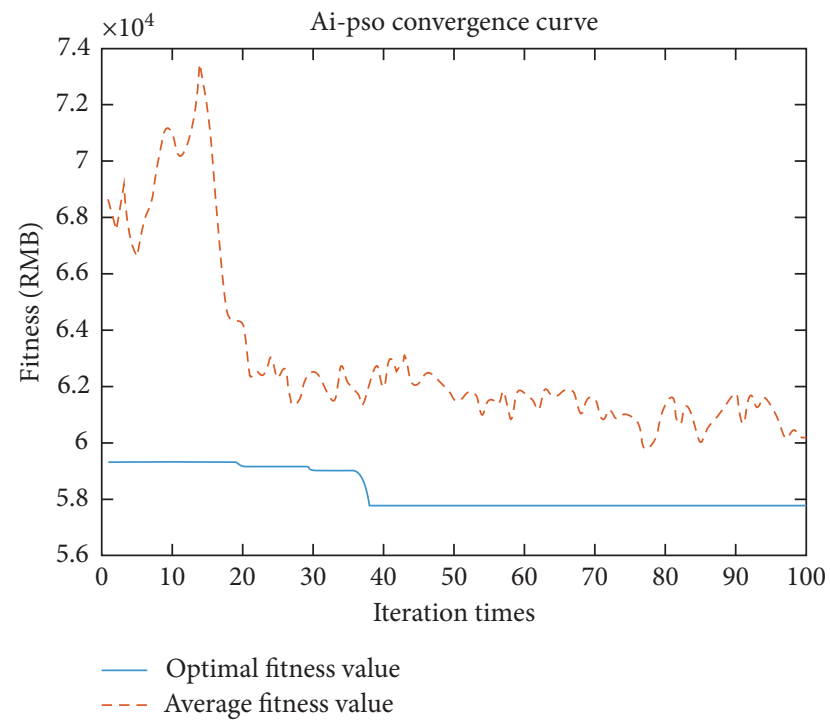

Figure 6: Convergence curve of AI-PSO in a static environment.

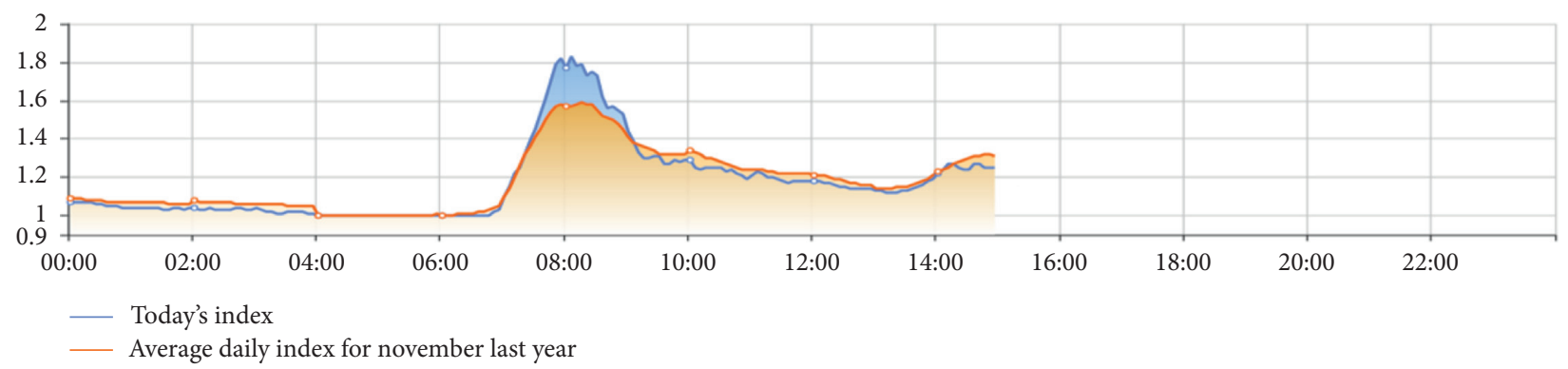

FIGURE 7: Real-time congestion index change.

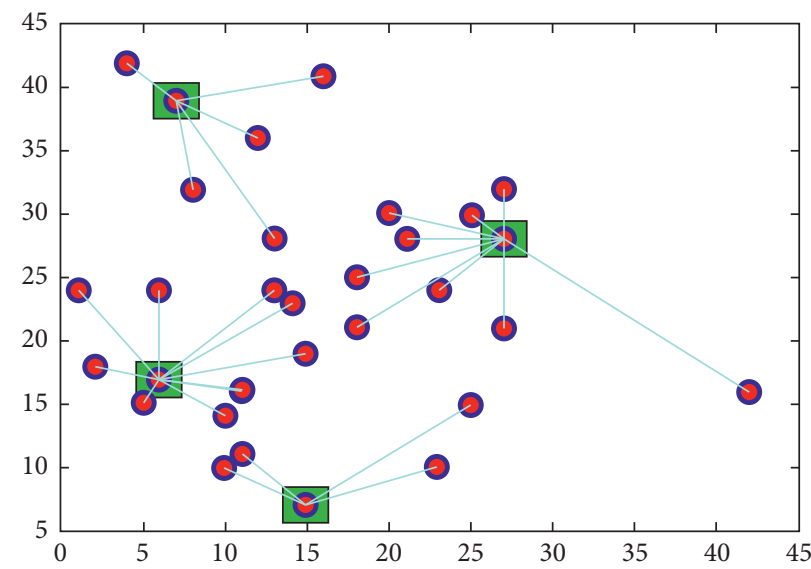

FIGURE 8: Customer ownership in a dynamic environment. 
TABLE 8: Service object information of suppliers and distribution centers in a dynamic environment.

\begin{tabular}{lc}
\hline Service outlets & Service object \\
\hline A & B16, B24, B27, B28 \\
B16 & B11, B16, B25, B26, B30 \\
B24 & B1, B5, B4, B9, B10, B18, B19, B24, B29 \\
B27 & B3, B6, B7, B8, B14, B17, B21, B23, B27, B31 \\
B28 & B2, B13, B15, B20, B22, B28 \\
\hline
\end{tabular}

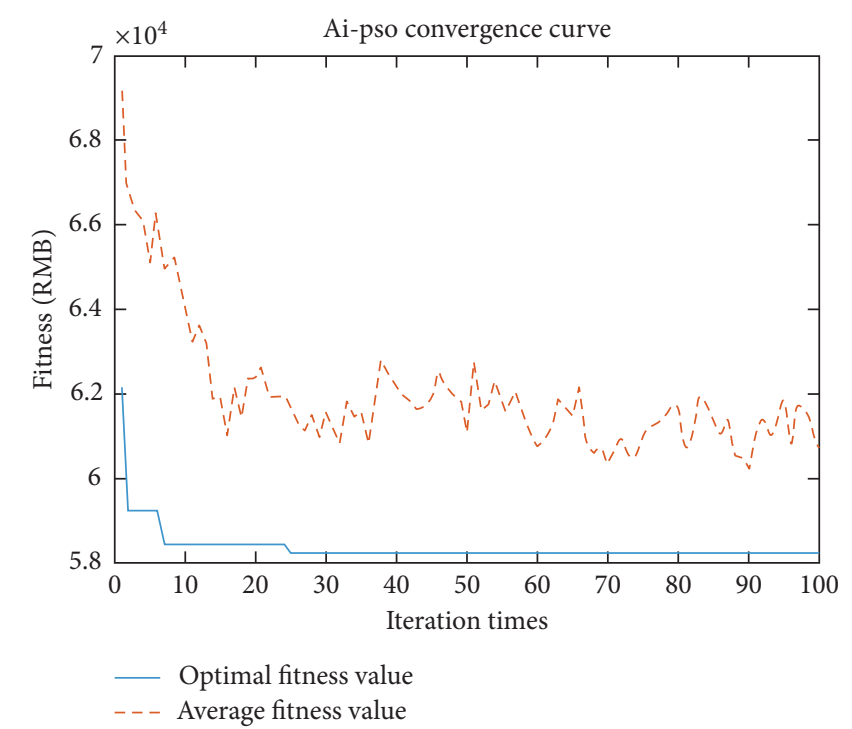

FIgURE 9: Convergence curve of AI-PSO in a dynamic environment.

TABLE 9: Cold chain transport path, distance, cost, and service reliability in a dynamic environment.

\begin{tabular}{lcccc}
\hline Service outlets & Shipping lines & Number and model of vehicle & Total cost & $\begin{array}{c}\text { Total distance } \\
\text { Service } \\
\text { reliability }\end{array}$ \\
\hline A & A-B27-B16-B24-B28-A & $5 \mathrm{~A}+1 \mathrm{C}+1 \mathrm{D}$ & 27371.62 & 80.802 \\
B16 & B16-B11-B25-B26-B30-B16 & $1 \mathrm{~B}$ & 5660.207 & 35.735 \\
B24 & B24-B18-B9-B10-B1-B5-B19-B4-B29-B24 & $1 \mathrm{~A}+1 \mathrm{~B}$ & 11325.199 & 80.736 \\
B27 & B27-B17-B6-B14-B21-B8-B3-B31-B23-B7-B27 & $2 \mathrm{~A}+1 \mathrm{D}$ & 7420.925 & 42.335 \\
B28 & B28-B20-B2-B13-B15-B22-B28 & $1 \mathrm{~B}+1 \mathrm{C}$ & 6323.274 & 0.74 .224 \\
\hline
\end{tabular}

TABLE 10: Three methods are used to calculate the results in the static environment.

\begin{tabular}{lcccccc}
\hline Algorithm & Total cost (yuan) & Total distance $(\mathrm{km})$ & Average service reliability & Running time $(\mathrm{s})$ & $\begin{array}{c}\text { Total number of } \\
\text { vehicles and models }\end{array}$ & $\begin{array}{c}\text { computation } \\
\text { complexity }\end{array}$ \\
\hline AI-PSO & 57613.908 & 282.039 & 0.8787 & 31.3470 & $8 \mathrm{~A}+3 \mathrm{~B}+2 \mathrm{C}+3 \mathrm{D}$ & $O\left(N^{2}\right)$ \\
GA-PSO & 57752.342 & 297.634 & 0.8401 & 33.2169 & $8 \mathrm{~A}+4 \mathrm{~B}+1 \mathrm{C}+2 \mathrm{D}$ & $O\left(N^{2}\right)$ \\
PSO & 56319.591 & 268.352 & 0.8319 & 40.1203 & $8 \mathrm{~A}+3 \mathrm{~B}+3 \mathrm{C}+2 \mathrm{D}$ & $O\left(N^{2}\right)$ \\
\hline
\end{tabular}

AI-PSO algorithm is 0.8787 . The test algorithm achieves the desired effect in the static environment.

As shown in Table 11, in the result of the two-layer model of the optimal path for vehicles under the GA-PSO algorithm with urban cold chain transportation cost as the objective function, the supplier needs to send five A cars, one $\mathrm{C}$ car, and one D car to carry out distribution for the four distribution centers. A total of three $\mathrm{A}$, three $\mathrm{B}$, one $\mathrm{C}$, and 1
D refrigerated transport vehicles are sent to the four distribution centers to deliver to 27 customers. Compared with GA-PSO and PSO, AI-PSO has no difference in vehicle selection. In terms of program running time, PSO and GAPSO take 41.0860 and 33.9370 s, respectively, to converge. AI-PSO takes 32.6719 s to converge, which is $20.48 \%$ more efficient than PSO. In terms of the total cost, GA-PSO has the highest total cost. AI-PSO has the lowest total cost, but its 
TABLE 11: Three methods are used to calculate the results under a dynamic environment.

\begin{tabular}{|c|c|c|c|c|c|c|}
\hline Algorithm & Total cost (yuan) & Total distance $(\mathrm{km})$ & Average service reliability & Running time (s) & $\begin{array}{c}\text { Total number of } \\
\text { vehicles and models }\end{array}$ & $\begin{array}{c}\text { Computation } \\
\text { complexity }\end{array}$ \\
\hline AI-PSO & 58101.23 & 283.832 & 0.8557 & 32.6719 & $8 \mathrm{~A}+3 \mathrm{~B}+2 \mathrm{C}+2 \mathrm{D}$ & $O\left(N^{2}\right)$ \\
\hline GA-PSO & 61433.02 & 306.947 & 0.8625 & 33.9370 & $8 \mathrm{~A}+3 \mathrm{~B}+2 \mathrm{C}+2 \mathrm{D}$ & $O\left(N^{2}\right)$ \\
\hline PSO & 58891.52 & 296.580 & 0.8466 & 41.0860 & $8 \mathrm{~A}+3 \mathrm{~B}+2 \mathrm{C}+2 \mathrm{D}$ & $O\left(N^{2}\right)$ \\
\hline
\end{tabular}

average service reliability is 0.8557 , which is between the values for PSO and GA-PSO. Although the average service reliability of the AI-PSO algorithm is not the highest, its total cost and total distance are the lowest. In the dynamic environment, the test algorithm also achieves the expected results, and the optimization scheme of the urban cold chain transportation path based on the AI-PSO algorithm is in line with the requirements of the economy and service reliability. The proposed algorithm could obtain better objective function values than the other two algorithms in both static and dynamic environments, which indicates that the proposed algorithm is feasible and effective under different optimization objectives.

Comparison of Tables 10 and 11 indicates that, with the AI-PSO algorithm, the total distribution cost in the static environment is 57613.908, and the total distribution cost in the dynamic environment is 58101.23 (an increase of $0.85 \%$ ). In the static and dynamic environments, the total distribution distance is 282.039 and 283.832 , respectively, with an increase of $0.67 \%$. The average service reliability of delivery in the static and dynamic environments is 0.8787 and 0.8557 , respectively, with a reduction of $2.62 \%$. This result shows that the time-varying network environment increases the distribution cost and reduces the average service reliability of cold chain logistics enterprises. To avoid the influence of time-varying network conditions on urban distribution, urban cold chain logistics and transportation enterprises should establish a reasonable service time for distribution service and a reasonable time window for cold chain demand customers. The calculation results show that taking the service reliability as the optimization goal increases the distribution cost to a certain extent, and the improvement of the service reliability of cold chain logistics enterprises will increase the distribution cost to some extent. Cold chain logistics enterprises should explore differentiated customer management in the delivery process based on the characteristics of customers and adopt hard time window service for important customers, and soft time window service for other less important customers.

\section{Conclusion}

According to the characteristics of strong time requirement of urban cold chain transportation, the main factor affecting the timeliness of cold chain is customer ownership. In this study, the time window constraint of trapezoidal fuzzy membership function is introduced in this study. The urban transit section involved time-varying factors in the process of change and led to a time delay problem. On the basis of service reliability maximization, an optimization model of urban cold chain transportation path under time-varying network conditions was constructed. AI-PSO was used to solve the model in two stages, namely, cold chain transportation path optimization under a static environment and real-time cold chain transportation optimization under a dynamic environment. After research, the following contributions have been made:

(1) The urban cold chain transport path model constructed in this paper involves a three-layer network, namely, supplier-distribution center-customer. Firstly, the location of the distribution center and the transportation path from the supplier to the distribution center are solved according to the model. Secondly, the customer attribution is determined and the transportation path from the distribution center to the customer is solved according to the customer attribution result.

(2) In the dynamic optimization stage, this paper used Baidu Map traffic big data (China Urban Traffic Congestion Index) to reflect the time-varying characteristics of urban road network and solved the urban cold chain transport path under the timevarying network conditions. Compared with the optimization of cold chain transportation path in the static environment, the results of the dynamic environment showed that the model could obtain the optimal distribution path with the lowest cost and adjusted the urban cold chain transportation path according to the real-time traffic information to avoid traffic congestion.

In the field of transportation, big data has begun to play an increasingly important role, and the role of big data in urban traffic management is gradually emerging. However, how to effectively distinguish and scientifically and rationally use data is a test of the wisdom and vision of decision makers. The model and algorithm presented in this paper can provide methodological support and a scientific reference to decision-makers of cold chain enterprises as they plan their transportation paths.

In fact, the traffic flow of urban roads is greatly different from that of expressways, and the measurement of vehicle arrival on the middle section of urban roads will also be seriously interfered by upstream signal lights and upstream bus stops. If the road is not long enough, it may also be disturbed by downstream traffic facilities. Wang [28] found that the number of vehicles arriving at a certain time interval on a road section was taken as a random number, and its 
statistical law could be described by three kinds of discrete distributions depending on different situations: Poisson distribution when traffic density is small. When the vehicle density is too large, it is binomial distribution. Negative binomial distribution is used for other cases and those affected by rush hour. However, for the convenience of solving the model in this paper, it is assumed that the time of the vehicle passing through section $(O, D)$ follows normal distribution $N\left(\mu_{O D}, \sigma\right)$, which is far from the actual situation. Future research in this field still needs efforts. Also this study does not consider the time-varying demand of customers, nor does it discuss the reverse logistics problems involving customers' returned goods and materials. Moreover, vehicle scheduling problems, such as the random timevarying characteristics of urban road networks, road section failure, and traffic avoidance based on real-time traffic information, are not included and thus need to be further studied.

\section{Data Availability}

The basic data required in this paper were obtained through public data collection and field research.

\section{Conflicts of Interest}

The authors declare that they have no conflicts of interest.

\section{Acknowledgments}

This work was funded by the following fund projects: NSFC funded project (71662011), Educational Research Project for Young and Middle Aged Teachers of Fujian Education Department (jas20228), Jiangxi Economic and Social Development Think Tank Project (19zk7), Jiangxi Social Science Planning Project (18gl7), Cultivation Project of "Tianyou plan" of East China Jiaotong University (2018xlty007), and Fujian Social Science Planning Project (fj2018b020).

\section{References}

[1] K. Govindan, A. Jafarian, R. Khodaverdi, and K. Devika, "Two-echelon multiple-vehicle location-routing problem with time windows for optimization of sustainable supply chain network of perishable food," International Journal of Production Economics, vol. 152, no. 2, pp. 9-28, 2014.

[2] C. Xiao, L. Y. Zhang, and T. Fei, "Research on bacteria foraging ant colony optimization algorithm for cold chain low carbon logistics distribution routing optimization," Mathematics in Practice and Theory, no. 21, pp. 100-109, 2017.

[3] K. Kang, J. Han, W. Pu et al., "Optimization research on cold chain distribution routes considering carbon emissions for fresh agricultural products," Computer Engineering and Applications, vol. 55, no. 2, pp. 259-265, 2019.

[4] C. D. Tarantilis and C. T. Kiranoudis, "A meta-heuristic algorithm for the efficient distribution of Perishable foods," Journal of Food Engineering, vol. 50, no. 1, pp. 1-9, 2001.

[5] P. Amorim and B. Almada-Lobo, "The impact of food perishability issues in the vehicle routing problem," Computers \& Industrial Engineering, vol. 67, no. 1, pp. 223-233, 2014.
[6] M. Rabbani, A. Farshbaf-Geranmayeh, and N. Haghjoo, "Vehicle routing problem with considering multi-middle depots for perishable food delivery," Uncertain Supply Chain Management, vol. 4, no. 3, pp. 171-182, 2016.

[7] Y. M. Zhang, Y. M. Li, and H. O. Liu, "Research on VRP optimization of multi-type vehicle cold-chain logistics with satisfaction constraint," Statistics \& Decision, vol. 4, pp. 176-181, 2019.

[8] H. J. Zheng, The Study of Vehicle Route Replanning for Cold Chain Logistics under Emergencies, Beijing Jiaotong University, Beijing, China, 2014.

[9] H. Lan, Q. F. He, and Z. Bian, "Distribution routing optimization of cold chain logistics with consideration of road traffic conditions," Journal of Dalian Maritime University, vol. 11, no. 41, pp. 67-74, 2015.

[10] Y. Deng and W. H. Zhu, "Research on scheduling optimization of military logistics distribution vehicles under time-varying conditions," Military Operations Planning and Systems Engineering, vol. 2, pp. 41-48, 2017.

[11] L. Wang and J. Lu, "A memetic algorithm with competition for the capacitated green vehicle routing problem," IEEE/CAA Journal of Automatica Sinica, vol. 6, pp. 516-526, 2019.

[12] S. L. Yang, H. W. Ma, and T. J. Gu, "Simulated annealing algorithm for vehicle scheduling with time windows under time-varying conditions," Operations Research Transactions, vol. 14, no. 3, pp. 84-90, 2010.

[13] Y. F. Li, J. Li, and Z. Y. Gao, "Traveling salesman problem in time varying network," Journal of Systems Engineering, vol. 25, no. 5, pp. 585-591, 2010.

[14] J. H. Li, "Time varying travelling planning problem for maximal utility," Chinese Journal of Management Science, vol. 19, no. 4, pp. 137-143, 2010.

[15] Z. Shi and Z. Fu, "Food cold chain distribution positioning with time window under time-varying network conditions optimization of transportation path," Application Research of Computers, vol. 1, pp. 183-189, 2013.

[16] J. F. Zhang and Z. H. Yang, "Research on optimization of multitemperature cold chain distribution path in time-varying road network environment," Journal of Chongqing Jiaotong University (Natural Science Edition), vol. 1, pp. 57-64, 2020.

[17] Z. H. Ding, Y. Zhou, and M. C. Zhou, "Modeling self-adaptive software systems by fuzzy rules and petri nets," IEEE Transactions on Fuzzy Systems, vol. 26, pp. 967-984, 2018.

[18] Y. H. Shen, W. Pedrycz, and X. M. Wang, "Approximation of fuzzy sets by interval type-2 trapezoidal fuzzy sets," IEEE Transactions on Cybernetics, vol. 50, pp. 4722-4740, 2018.

[19] T. Jin, X. Yang, H. Xia et al., "Reliability index and option pricing formulas of the first hitting time model based on the uncertain fractional-order differential equation with Caputo type," Fractals, vol. 1, 2020.

[20] W. Deng, J. J. Xu, X. Z. Gao et al., “An enhanced MSIQDE algorithm with novel multiple strategies for global optimization problems," IEEE Transactions on Systems, Man, and Cybernetics: Systems, vol. 19, pp. 1231-1256, 2020.

[21] J. Li, J. Q. Zhang, C. J. Jiang et al., "Composite particle swarm optimizer with historical memory for function optimization," IEEE Transactions on Cybernetics, vol. 45, no. 10, pp. 2350$2363,2017$.

[22] W. Dong and M. C. Zhou, "A supervised learning and control method to improve particle swarm optimization algorithms," IEEE Transactions on Systems, Man, and Cybernetics: Systems, vol. 47, no. 7, pp. 1149-1159, 2017.

[23] J. Zhang, X. Zhu, Y. Wang, and M. Zhou, "Dual-environmental particle swarm optimizer in noisy and noise-free environments," 
IEEE Transactions on Cybernetics, vol. 49, no. 6, pp. 2011-2021, 2019.

[24] T. Jin, H. Xia, and H. Chen, "Optimal control problem of the uncertain second-order circuit based on first hitting criteria," Mathematical Methods in the Applied Sciences, vol. 8, 2020.

[25] Y. Song, D. Wu, W. Deng et al., "MPPCEDE: multi-population parallel co-evolutionary differential evolution for parameter optimization," Energy Conversion and Management, vol. 228, Article ID 113661, 2021.

[26] N. J. Y. Chen, L. Li, and Y. Luo, "Bidirectional clustering algorithm of quantum particle swarm optimization based on diversity selection," Computer Engineering and Applications, vol. 54, no. 9, pp. 42-46, 2018.

[27] G. Peng, Y. W. Fang, and W. S. Peng, "Multi-objective particle optimization algorithm based on sharing learning and dynamic crowding distance," Optik, vol. 27, no. 9, pp. 5013-5020, 2016.

[28] D. H. Wang, Traffic Flow theory, People's Communications Press, Beijing, China, 2002. 